\title{
Charmonium-nucleon interactions from the time-dependent HAL QCD method
}

\author{
Takuya Sugiura ${ }^{1, \star}$, Yoichi Ikeda ${ }^{1}$, and Noriyoshi Ishii ${ }^{1}$ \\ ${ }^{1}$ Research Center for Nuclear Physics (RCNP), Osaka University, Osaka 567-0047, Japan
}

\begin{abstract}
The charmonium-nucleon effective central interactions have been computed by the time-dependent HAL QCD method. This gives an updated result of a previous study based on the time-independent method, which is now known to be problematic because of the difficulty in achieving the ground-state saturation. We discuss that the result is consistent with the heavy quark symmetry. No bound state is observed from the analysis of the scattering phase shift; however, this shall lead to a future search of the hidden-charm pentaquarks by considering channel-coupling effects.
\end{abstract}

\section{Introduction}

Properties of hadrons having more complex structure than those of the standard three-quark baryons or the quark-antiquark mesons, called exotic hadrons, have not been established yet. Recently, the LHCb collaboration have reported the observation of two hidden-charm pentaquark candidates, $P_{c}(4380)$ and $P_{c}(4450)$, in the $J / \psi p$ invariant mass distribution from the weak decay of the $\Lambda_{b}^{0} \rightarrow J / \psi p K^{-}$ [1]. The fit with the Breit-Wigner parameterization shows the masses and widths as $M_{P_{c}(4380)}=$ $205 \pm 18 \pm 86 \mathrm{MeV}, \Gamma_{P_{c}(4380)}=205 \pm 18 \pm 86 \mathrm{MeV}, M_{P_{c}(4450)}=4449.8 \pm 1.7 \pm 2.5 \mathrm{MeV}$, and $\Gamma_{P_{c}(4450)}=$ $39 \pm 5 \pm 19 \mathrm{MeV}$. Three acceptable spin-parity combinations $\left(3 / 2^{-}, 5 / 2^{+}\right),\left(3 / 2^{+}, 5 / 2^{-}\right),\left(5 / 2^{+}, 3 / 2^{-}\right)$ are also obtained.

A lot of theoretical works have been done to reveal the structure of the $P_{c}$ 's [2-6]. These results do not give a consistent answer about what the reported $P_{c}$ resonances actually are like, and even the existence of such resonances. We believe this situation originates from the lack of knowledge of interactions between charmed hadrons. Shown in figure 1 is a diagram for the decay $\Lambda_{b} \rightarrow P_{c} K$. The observed peaks of $P_{c}$ must couple to the $J / \psi N$ were they really resonances. Therefore, it would be desirable to consider the 2-body scattering without the spectator $K$ to study $P_{c}$. Obviously such an experiment is unaccessible, and another approach is in demand.

To settle the above issue, we aim at studying the structures of the $P_{c}$ 's via the lattice QCD simulations without requiring further experimental data. We employ the potential approach developed by the HAL QCD collaboration[7]. The method, which we shall call the (time-independent) HAL $Q C D$ method, utilizes the spatial part of hadron correlators, called the Nambu-Bethe-Salpeter (NBS) amplitude. The long-distance asymptotic behavior of the NBS amplitude is identical to that of a scattered wave function in quantum mechanics; therefore, by solving the Schrödinger equation for the potential with such an amplitude as input, we can be sure that the potential is faithful to the QCD

${ }^{\star}$ Speaker, e-mail: sugiura@rcnp.osaka-u.ac.jp 
$\mathrm{S}$ matrix. It has already been applied to a variety of systems, including the nucleon-nucleon (NN), nucleon-hyperon (NY), YY, and NNN interactions [8]. The above method relies on the ground-state saturation in correlation functions. However, the ground-state saturation has turned out difficult to achieve in actual calculations. To avoid this problem, the HAL QCD collaboration has introduced another method, called the time-dependent method [9]. In the time-dependent method, we assume that correlation functions are dominated not only by the ground state but by the elastic states. By solving a time-dependent Schrödinger-like equation, we calculate the same potential as in the original method. Since the elastic-state saturation is much easier to achieve than the ground-state saturation, the timedependent method should always be preferred. Moreover, the HAL QCD method is straightforwardly applied to coupled-channel systems [8]. Since channel-coupling is expected to be crucial to study the origin of the peak corresponding to the $P_{c}$ 's, this feature is quite helpful.

In the present study, we have set out on our coupled-channel analysis from the $J / \psi N$ and $\eta_{c} N$ single-channel interactions. We focus on the S-wave angular momentum, so we have three channels: $J / \psi N\left(J^{P}=1 / 2^{-}\right), J / \psi N\left(J^{P}=3 / 2^{-}\right)$, and $\eta_{c} N\left(J^{P}=1 / 2^{-}\right)$. These channels are a part of the coupled channels that can couple to $P_{c}$. The $J / \psi N\left(J^{P}=3 / 2^{-}\right)$in particular, is the lowest-lying channel of the five $\mathrm{S}$-wave meson-baryon channels in Figure 1. We calculate the effective central potential for each of the three channels, so that the couplings to the other meson-baryon channels, to the channels with higher angular momentum, and between $J / \psi N$ and $\eta_{c} N$ in the $J=1 / 2$ case, are all integrated out. The charmonium-nucleon effective central interactions have already been studied with the quenched lattice QCD in Ref. [10]. They found no bound state between $\eta_{c} N$ nor $J / \psi N$. In contrast, authors in Ref. [11] have reported contradictory results from the direct method: the $\eta_{c} N$ system has a deeply bound state with binding energy of $19.8 \mathrm{MeV}$. In this way, there exists a conflict between the HAL QCD potential method and the direct method as in the NN sector where the ground state saturation plays a key role [12]. Since the authors of Ref. [10] have employed the old-fashioned HAL QCD method (i.e. the time-independent method) which can be afflicted with the problem of the ground state saturation, we shall improve their results by employing the new one (i.e. the time-dependent HAL QCD method) to avoid the problem. Moreover, we shall see that the comparison of our results for the two spin assignments of the $J / \psi N$ gives an implication of the heavy quark spin symmetry.

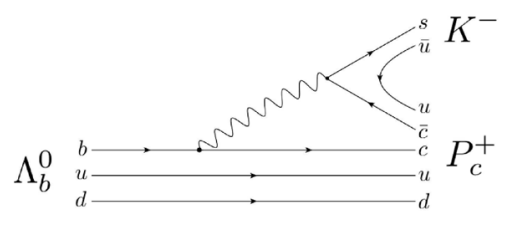

Figure 1. Feynman diagram for the $\Lambda_{b}^{0} \rightarrow P_{c}^{+} K^{-}$decay.

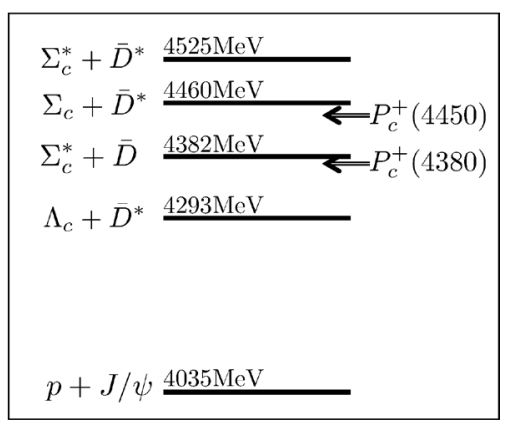

Figure 2. The five S-wave meson-baryon channels that possibly couple to the pentaquarks with $J^{P}=3 / 2^{-}$. 


\section{Method}

We start from computing a normalized four-point correlation function $R$ of each charmonium-nucleon system,

$$
R(\vec{r}, t) \equiv\left\langle 0\left|T N(\vec{x}+\vec{r}, t) \phi(\vec{x}, t) \overline{\mathcal{J}}\left(t_{0}=0\right)\right| 0\right\rangle / e^{-\left(m_{N}+m_{\phi}\right) t},
$$

where $N(\vec{x}, t)$ denotes an interpolating operator for the nucleon, $\phi(\vec{x}, t)$ for the charmonium (either $J / \psi$ or $\eta_{c}$ ), and $\overline{\mathcal{T}}\left(t_{0}\right)$ the corresponding wall-source operator. In actual lattice calculations, the exponential factor involving the nucleon mass $m_{N}$ and the charmonium mass $m_{\phi}$ is replaced by the single-hadron correlators. The non-local potential $U\left(\vec{r}, \vec{r}^{\prime}\right)$ satisfies the Schrödinger-like equation at $t$ large enough for the elastic-state saturation

$$
\left(-\frac{\partial}{\partial t}+\frac{\vec{\nabla}^{2}}{2 \mu}\right) R(\vec{r}, t)=\int d^{3} \vec{r}^{\prime} U\left(\vec{r}, \vec{r}^{\prime}\right) R\left(\vec{r}^{\prime}, t\right),
$$

where $\mu=1 /\left(1 / m_{N}+1 / m_{\phi}\right)$ is the reduced mass. We neglect the relativistic effects, which in principle appear as higher-order time derivatives on the left-hand side, since they are much smaller than the other terms. The non-locality of the potential $U\left(\vec{r}, \vec{r}^{\prime}\right)$ can be well-tamed by the derivative expansion, such that the scattering phase shift computed from the expanded potential approaches the exact one order by order [13]. By taking the leading order term of the derivative expansion, $U\left(\vec{r}, \vec{r}^{\prime}\right) \simeq V(r) \delta^{3}(\vec{r}-$ $\vec{r}^{\prime}$ ), we get the effective central potential

$$
V_{\mathrm{eff}}(r)=-\frac{\partial_{t} R(\vec{r}, t)}{R(\vec{r}, t)}+\frac{1}{2 \mu} \frac{\vec{\nabla}^{2} R(\vec{r}, t)}{R(\vec{r}, t)} \equiv V_{t}(r)+V_{\nabla}(r) .
$$

Note that $V_{\text {eff }}(r)$ does not depend on $t$ although the $R$ correlator does. This is true when the elasticstate saturation is achieved and the local potential well approximates the non-local kernel $U\left(\vec{r}, \vec{r}^{\prime}\right)$. Furthermore, when the ground-state saturation is achieved, $V_{t}(r)$ is reduced to just a constant, $V_{t}(r)=\epsilon$ with $\epsilon$ independent of both $t$ and $r$. In other words, when $V_{t}(r)$ is dependent on $r$, the time-independent method will presumably fail.

\section{Simulation Setup}

We employ the $2+1$ flavor full QCD gauge configurations by the CP-PACS and JLQCD Collaborations [14]. They are generated with the renormalization-group improved gauge action and a nonperturbatively $O(a)$ improved clover quark action at $\beta=1.83$ (corresponding to the lattice spacing of $a=0.1209 \mathrm{fm}[15])$ on a $16^{3} \times 32$ lattice, so that the spatial volume is $(1.93 \mathrm{fm})^{3}$. The heavy mass of charm quarks may bring large discretization errors, for which the relativistic heavy quark (RHQ) action [16] should be employed. However, in this present study, we use the clover action for all quarks. The hopping parameter for the light (up and down) quarks and the strange quark are set to $\kappa_{u d}=0.13760$ and $\kappa_{s}=0.13710$, respectively. For the charm quark, the value $\kappa_{c}=0.11660$ is determined by fitting the $1 / \kappa$ vs $m_{\mathrm{PS}}^{2}$ plot to reproduce the physical $\eta_{c}$ mass of $2983 \mathrm{MeV}$ on this lattice.

The Dirichlet boundary condition is imposed at $t-t_{0}=26$ to prevent inverse propagation. In the spatial directions we imposed the periodic boundary condition. We average the results from 16 source positions $t_{0}$ to reduce statistical error. The spatial correlation is averaged based on the 48 octahedral group transformations. 


\section{Results and Discussion}

In Figure 3, we show the effective masses of $N, \eta_{c}$, and $J / \psi$ extracted from single-hadron correlation functions. We see plateaux in all those plots. We have performed a single exponential fit to each of the single-hadron correlators. The resulting masses are $m_{N}=1816 \mathrm{MeV}(t=8-14), m_{\eta_{c}}=2996 \mathrm{MeV}$ $(t=15-18)$, and $m_{J / \psi}=3089 \mathrm{MeV}(t=16-19)$, where the time slices used for the fit are shown in the parentheses.
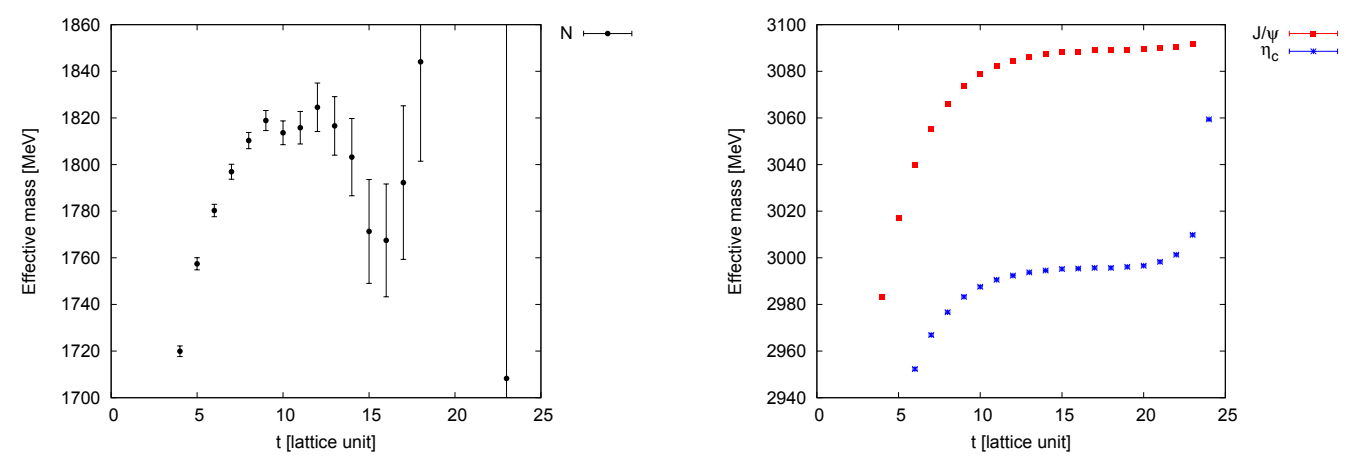

Figure 3. The effective masses of (left) nucleon, (right,blue) $\eta_{c}$, and (right,red) $J / \psi$.

Figure 4 shows our results of the effective central potentials at $t=12$. They are all attractive overall, and are quite similar quantitatively. Especially, the two total spin assignments of $J / \psi N$ have very small difference, which differ in the spin direction of $J / \psi$ relative to that of $N$, when the $S$ wave angular momentum considered. This may be because of the following reason. According to the heavy quark spin symmetry [17], light quarks and soft gluons see a heavy quark as a static color source, since they cannot change the velocity or flip the spin of the heavy quark. Thus the dynamics is independent of the heavy quark spin, as the difference is suppressed by $O\left(1 / m_{h}\right)$ for heavy quark with mass $m_{h}$. This picture seems to be applicable to the two spin assignments of the $J / \psi N$. The $\eta_{c} N$ potential has a little weaker attraction than the $J / \psi N$ potentials. This might be because of the lighter mass of $\eta_{c}$ than of $J / \psi$ by $93 \mathrm{MeV}$. The authors of Ref. [10] have also observed a similar tendency between the $\eta_{c} N$ and spin-averaged $J / \psi N$ potentials. They have discussed that the difference in the reduced masses is too small to account for the $O(10) \%$ difference in the potentials, and thus it is caused by different structures of $\eta_{c}$ and $J / \psi$. We cannot draw a definite conclusion either; however we remark that the similarity in the behavior of the charmonium-nucleon interactions, and yet the small difference between the $\eta_{c} N$ and $J / \psi N$, are compatible with the heavy quark symmetry.

In figure 5, we show the $J / \psi N(J=1 / 2)$ potentials extracted at $t=12-15$, where the filled areas express statistical error bars. We see all the results agree within the errors. Therefore we conclude that the elastic-state saturation is achieved and the derivative expansion is converged at the lowest order at $t=12$. The same check has been done to the other two potentials, and they are found qualitatively similar to this case.

Notice that the $R$ correlator in Eq. (1) is normalized by a single exponential factor, so that we assume the ground-state saturation of the $J / \psi$ and $N$ single-hadron correlators. As can be seen in figure 3, however, $t=12$ is a little too small for the single-charmonium (both $\eta_{c}$ and $J / \psi$ ) correlators to achieve the ground-state saturation. It results in $\sim 3 \mathrm{MeV}$ deviation in the effective masses of the charmonia at $t=12$ and those extracted from the plateaux in $t>15$. Nevertheless, the deviation is 


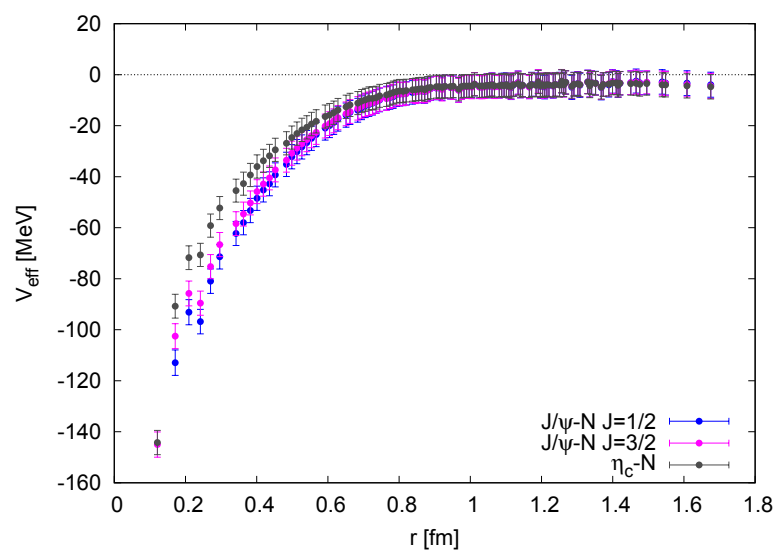

Figure 4. Effective central potentials at $t=12$ of (black) $\eta_{c} N$, (blue) $J / \psi N$ with $J=1 / 2$, and (magenta) $J / \psi N$ with $J=3 / 2$.

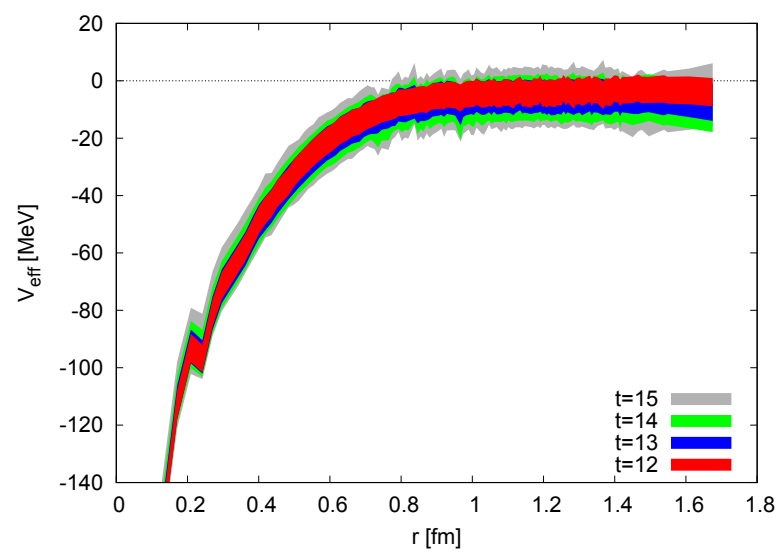

Figure 5. The $J / \psi N(J=1 / 2)$ potentials extracted at different time slices, $t=12-15$.

of $\sim 0.1 \%$ relative to the total masses of $\sim 3000 \mathrm{MeV}$, so that it affects little to the potentials, as is actually seen in Figure 5.

Figure 6 shows each term of the $J / \psi N(J=1 / 2)$ potential in Eq. (3), depicted separately. The second term $V_{\nabla}(r)$ gives dominant contribution to the short-distance attraction, and it behaves repulsively for $r>0.6$. The first term $V_{t}(r)$ is attractive overall with smaller strength at short distances than $V_{\nabla}(r)$. At large distances, the small repulsion of $V_{\nabla}(r)$ and the small attraction of $V_{t}(r)$ cancel and give the totally attractive and short-ranged $V_{\text {eff }}(r)$. Notice that $V_{t}(r)$ is dependent on $r$; it means that the ground-state saturation of the four-point correlation function is not achieved. The time-dependent method gives a desired potential, as $V_{\text {eff }}(r)$ is naturally connected to outside of its range. 


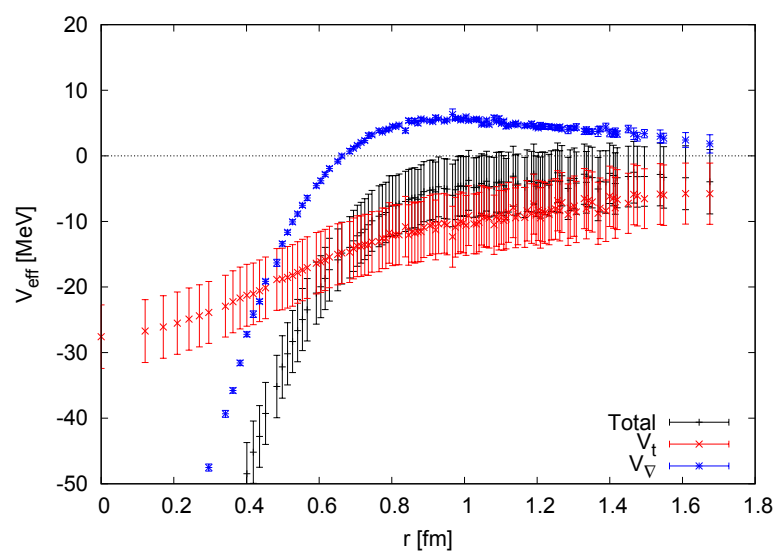

Figure 6. Each term of the $J / \psi N(J=1 / 2)$ potential in Eq. (3): (red) $V_{t}(r)$, (blue) $V_{\nabla}(r)$, and (black) $V_{\text {eff }}(r)$ at $t=12$.

We have performed a fit of the potentials in Figure 4 with two Gaussian functions. Then by solving the S-wave radial Schrödinger equation

$$
\left\{\frac{1}{2 \mu r^{2}} \frac{d}{d r}\left(r^{2} \frac{d}{d r}\right)+E\right\} R(r ; E)=V(r) R(r ; E)
$$

we obtain the scattering phase shift $\delta(E)$ from the asymptotic form of $R(r ; E)$,

$$
R(r ; E) \sim \frac{i}{2}\left(h_{0}^{(-)}(k r)-e^{2 i \delta(E)} h_{0}^{(+)}(k r),\right)
$$

where $h_{0}^{( \pm)}(z)$ are the spherical Riccati-Hankel functions with $l=0$.

Figure 7 shows the result of the phase shift of $J / \psi N(J=1 / 2)$. We see that the interaction is attractive, but not strong enough to have bound states. Moreover we have performed the effectiverange expansion $k \cot (\delta)=1 / a+r k^{2} / 2$ to obtain the scattering length $a$ and the effective range. At $t=12$, the results are: $a=0.68 \pm 0.44 \mathrm{fm}$ and $r=1.04 \pm 0.03 \mathrm{fm}$ for $J / \psi N(J=1 / 2), a=0.63 \pm 0.42 \mathrm{fm}$, $r=1.11 \pm 0.03 \mathrm{fm}$ for $J / \psi N(J=3 / 2)$, and $a=0.44 \pm 0.34 \mathrm{fm}, r=1.33 \pm 0.06 \mathrm{fm}$ for $\eta_{c} N$.

\section{Conclusions}

We have computed the S-wave effective central potentials of the $J / \psi N\left(J^{P}=1 / 2^{-}\right.$and $\left.3 / 2^{-}\right)$and $\eta_{c} N$. We have shown that our results are qualitatively similar to the previous results by Kawanai and Sasaki, where the time-independent method is employed. However, we have confirmed that the time-dependent method is crucial to extract a reliable potential, as the ground-state saturation is not achieved at a moderate time. Our results show that all the charmonium-nucleon potentials are quite similar, and this is naturally understood through the heavy quark symmetry. We have not observed any bound states as shown in the phase shift analysis. However, inclusion of the other channels might result in emergence of resonance states, which may correspond to the $P_{c}$ resonances. The analysis with coupled channels and the use of the RHQ action are currently in progress. 


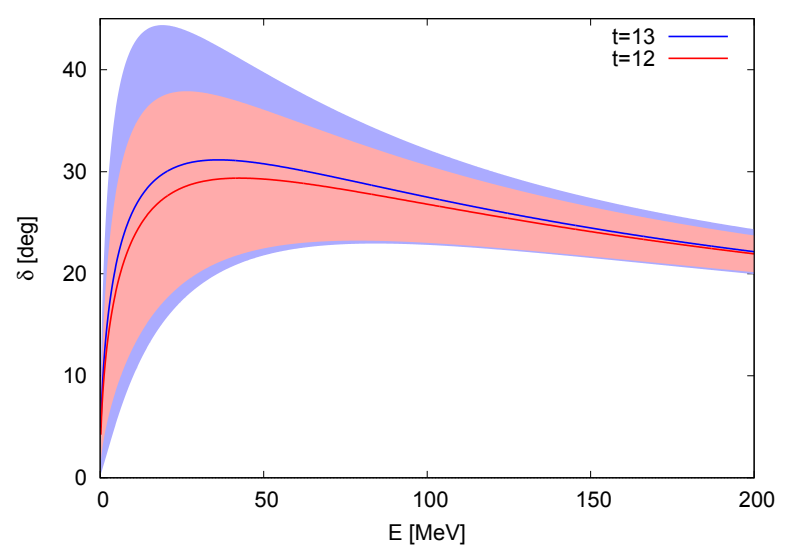

Figure 7. The scattering phase shift of the $J / \psi N(J=1 / 2)$ at $t=12$ and 13 .

\section{Acknowledgements}

This work is supported by Japan Society for the Promotion of Science KAKENHI Grands No. JP25400244 and by Ministry of Education, Culture, Sports, Science and Technology as "Priority Issue on Post-K computer" (Elucidation of the Fundamental Laws and Evolution of the Universe) and Joint Institute for Computational Fundamental Science. We thank CP-PACS and JLQCD Collaborations [15] and ILDG/JLDG [18] for the 2+1 flavor QCD gauge configurations. This work is in part based on Bridge++ code [19].

\section{References}

[1] R. Aaij at al. [LHCb Collaboration], Phys. Rev. Lett. 115, 072001 (2015).

[2] J. J. Wu, R. Molina, E. Oset and B. S. Zou, Phys. Rev. Lett. 105, 232001 (2010) doi:10.1103/PhysRevLett.105.232001 [arXiv:1007.0573 [gull-th]].

[3] R. Chen, X. Liu, X. Q. Li and S. L. Zhu, Phys. Rev. Lett. 115, no. 13, 132002 (2015).

[4] H. X. Chen, W. Chen, X. Liu, T. G. Steele and S. L. Zhu, Phys. Rev. Lett. 115, no. 17, 172001 (2015).

[5] L. Maiani, A. D. Polosa and V. Query, Phys. Lett. B 749, 289 (2015).

[6] F. K. Guo, U. G. Meißner, W. Wang and Z. Yang, Phys. Rev. D 92, no. 7, 071502 (2015).

[7] N. Ishii, S. Aoki and T. Hazed, Phys. Rev. Lett. 99, 022001 (2007).

[8] S. Aoki et al. [HAL QCD Collaboration], PTEP 2012, 01 A105 (2012).

[9] N. Ishii et al. [HAL QCD Collaboration], Phys. Lett. B 712, 437 (2012).

[10] T. Kawanai and S. Sasaki, Phys. Rev. D 82, 091501 (2010).

[11] S. R. Beane, E. Chang, S. D. Cohen, W. Detmold, H.-W. Lin, K. Orginos, A. Parreño and M. J. Savage, Phys. Rev. D 91, no. 11, 114503 (2015).

[12] T. Iritani et al., Phys. Rev. D 96, no. 3, 034521 (2017).

[13] T. Sugiura, N. Ishii and M. Oka, Phys. Rev. D 95, no. 7, 074514 (2017).

[14] See "http://www.jldg.org/ildg-data/CPPACS+JLQCDconfig.html" for more detail.

[15] T. Ishikawa et al. [JLQCD Collaboration], Phys. Rev. D 78, 011502 (2008).

[16] S. Aoki, Y. Kuramashi and S. i. Tominaga, Prog. Theor. Phys. 109, 383 (2003).

[17] N. Isgur and M. B. Wise, Adv. Ser. Direct. High Energy Phys. 10, 234 (1992). 
[18] http://plone.jldg.org/wiki/index.php/Main_Page; http://www.jldg.org/.

[19] http://bridge.kek.jp/Lattice-code/ 